\title{
Estratificação vertical de abelhas Euglossina (Hymenoptera, Apidae) em uma área de Mata Atlântica, Paraíba, Brasil
}

\author{
Celso F. Martins \& Alysson K. P. de Souza
}

Departamento de Sistemática e Ecologia, Centro de Ciências Exatas e da Natureza, Universidade Federal da Paraíba. 58059-900 João Pessoa, Paraíba, Brasil.E-mail: cmartins@dse.ufpb.br; alysson@dse.ufpb.br

\begin{abstract}
Vertical stratification of Euglossina Bees (Hymenoptera, Apidae) in an area of the Atlantic Rainforest, Paraíba State, Brazil. There are few studies regarding vertical distribution of insects, mainly of Hymenoptera Apiformes. The goal of this study was to verify if vertical stratification exists among the species of Euglossina in Atlantic Rainforest areas, trough the comparison of the richness, abundance and diversity in two strata, sub-forest $(1.5 \mathrm{~m})$ and canopy $(10-12 \mathrm{~m})$. For that, monthly collections where carried out in the Guaribas Biological Reserve from May to December of 2002. Six traps containing artificial fragrances: eugenol, eucalyptol, skatol, beta ionone, benzyl acetate and vanillin, distributed in two strata were used. A total of 1,151 individuals belonging to 11 species and three genera of Euglossina was collected. The abundance and diversity were higher in the sub-forest stratum in absolute number and richness was significantly higher compared with the canopy.

KEY WORDS. Abundance, canopy, Euglossa, Eulaema, richness.
\end{abstract}

RESUMO. Existem poucos estudos sobre distribuição vertical de insetos, principalmente de Hymenoptera Apiformes. O objetivo do presente estudo foi verificar se existe estratificação vertical entre as espécies de Euglossina em áreas de Mata Atlântica, através da comparação da riqueza, abundância e diversidade em dois estratos, subbosque $(1,5 \mathrm{~m})$ e copa $(10-12 \mathrm{~m})$. Para isso, foram realizadas coletas mensais na Reserva Biológica Guaribas, de maio a dezembro de 2002, utilizando seis armadilhas contendo fragrâncias artificiais: eugenol, eucaliptol, escatol, beta ionona, acetato de benzila e vanilina, distribuídas nos dois estratos. Foram coletados 1.151 indivíduos pertencentes a 11 espécies e três gêneros de Euglossina. No estrato de sub-bosque a abundância e a diversidade foram maiores, em números absolutos, e a riqueza foi significativamente maior que no estrato de copa.

PALAVRAS CHAVE. Abundância, copa, Euglossa, Eulaema, riqueza.

A subtribo Euglossina possui 175 espécies descritas, formada por abelhas de tamanho médio a grande, as quais possuem, geralmente, um colorido metálico vivo (Silveira et al. 2002). KIMSEY (1982) reconheceu para este grupo cinco gêneros, dentre eles, três de vida livre (Eufriesea Cockerell, 1909; Eulaema Lepeletier, 1841 e Euglossa Latreille, 1802) e dois cleptoparasitas: (Aglae Lepeletier \& Serville, 1825 e Exaerete Hoffmannsegg, 1817). O grupo ocorre exclusivamente na região Neotropical, entre $30^{\circ}$ de latitude Norte e $32^{\circ}$ de latitude Sul, com algumas espécies se distribuindo além dos trópicos (Michener 2000, RebêLo 2001).

Os machos das espécies de Euglossina destacam-se por coletarem substâncias odoríferas nas flores de diversas plantas, entre elas: Orchidaceae, Araceae, Myrtaceae, Leguminosae, Bignoniaceae, Gesneriaceae, Solanaceae e Euphorbiaceae, além de fontes extra-florais (Dressler 1982, Ackerman 1983, Williams \& WhitTen 1983). A função dessas substâncias odoríferas ainda não está esclarecida. Provavelmente, estas substâncias são misturadas para formar um "bouquet" espécie-específico (Eutz et al. 1999) e usadas para atrair outros machos num comportamento denominado "lek" (Dodson 1966, KimSey 1980, DresSLER 1982, SCHEMSKE \& Lande 1984, Stern 1991, Peruquetti 2000, Roubik \& Hanson 2004). Inúmeros estudos foram realizados com Euglossina em ambientes de Mata Atlântica no Brasil (e.g. WitTMAnN et al. 1988, 1989, Silveira \& Cure 1993, Rebêlo \& Garófalo 1991, 1997, Peruquetti et al. 1999, Bezerra \& Martins 2001, Tonhasca et al. 2002) e em áreas de domínio da Amazônia (e.g. Braga 1976, Powel \& Powell 1987, Becker et al. 1991, Morato 1994, Morato et al. 1992, Rebêlo \& Silva 1999). Entretanto, poucos estudos como Oliveira \& CAMPos (1996) para a Amazônia brasileira, RoubiK (1993) e Otero \& Sallenave (2003) para florestas do Panamá e Amazônia peruana, respectivamente, analisaram a composição faunística e a diversidade de Euglossina em diferentes estratos da vegetação.

Em regiões tropicais, estima-se que a fauna de artrópodes na copa seja no mínimo duas vezes mais rica do que no solo (ERWIN 1982). Para explicar as razões de tais preferências, SALMAH et al. (1990) sugerem que as condições micro-climáticas podem 
variar muito entre o interior do sub-bosque e as margens das copas. Segundo esses autores, os sub-bosques se caracterizam por apresentar menores temperaturas, luminosidade, correntes de ar e alta umidade, enquanto que nas copas ocorre o contrário. Portanto, esses gradientes micro-climáticos produziriam micro-habitats nos quais diferentes animais, inclusive abelhas, se adaptariam. Oliveira \& CAMPos (1996) sugerem que a estratificação vertical das Euglossina também pode estar relacionada à estratificação das orquídeas, com seu hábito epífito, que, segundo BRAGA (1987), estão situadas em diferentes gradientes verticais.

O objetivo deste trabalho foi comparar riqueza, abundância e diversidade da comunidade de Euglossina em diferentes estratos da vegetação de Mata Atlântica em uma Reserva Biológica no estado da Paraíba, região Nordeste do Brasil. Vale ressaltar que este é o primeiro estudo sobre estratificação vertical de Euglossina nesse ecossistema.

\section{MATERIAL E MÉTODOS}

\section{Área de estudo}

A Reserva Biológica Guaribas localiza-se nos Municípios de Mamanguape e Rio Tinto $\left(06^{\circ} 44^{\prime} \mathrm{S}, 35^{\circ} 08^{\prime} \mathrm{W}\right)$, no litoral Norte do estado da Paraíba, distando cerca de $80 \mathrm{~km}$ de João Pessoa. A área da Reserva foi inicialmente utilizada para um assentamento do INCRA, e desde a sua criação (25 de dezembro de 1990), não possui uma estrutura normal de Reserva. Apresenta uma área total de 4.321 ha divididos em três áreas separadas, recortadas por aglomerados urbanos, canaviais e culturas de subsistência dos assentados (IвAма 1995). O presente estudo foi realizado na maior área da Reserva com 3378,2 ha, localizada no município de Mamanguape. O clima da região é do tipo As' de Köppen, quente e úmido, com uma estação seca na primavera - verão e outra chuvosa no outono - inverno (GoverNo DO Estado da Paraíba 1985). Apresenta uma precipitação pluviométrica média anual de $1800 \mathrm{~mm}$ e as médias mensais de temperatura oscilam entre $24-26^{\circ} \mathrm{C}$ (IвAMA 1995).

A área da Reserva possui dois tipos de fitofisionomia principais: Savana Arbórea Aberta, um tipo de Cerrado, conhecida no nordeste como Tabuleiro (Prates et al. 1981, Oliveira-Filho \& CARvalHo 1993) e Floresta Estacional Semidecídua, caracterizada por uma formação florestal com espécies típicas da Mata Atlântica e elementos amazônicos (Darrault \& Schlindwein 2002). Esta floresta encontra-se em processo de regeneração, caracterizando-se por uma vegetação secundária, com dossel atingindo em média de $12-15 \mathrm{~m}$ de altura. Recentemente, a Reserva Biológica Guaribas foi incluída na área Mamanguape/ Baía Formosa (Paraíba/Rio Grande do Norte), definida como de extrema importância biológica e área de ação prioritária para a conservação e manejo da Mata Atlântica (MMA 2000).

\section{Amostragem das Abelhas}

Foram realizadas coletas mensais na área de floresta da Reserva, de maio a dezembro de 2002, das 6:00 às 14:00 horas, utilizando seis fragrâncias artificiais: eugenol, eucaliptol, escatol, beta ionona, acetato de benzila e vanilina.

As armadilhas para a coleta das abelhas foram confeccionadas usando garrafas tipo PET, conforme Bezerra \& Martins (2001), e distribuídas igualmente em dois estratos, no sub-bosque $(1,5 \mathrm{~m}$ de altura) e na copa (10-12m de altura), num total de 12 armadilhas. Cada uma foi separada linearmente por $5 \mathrm{~m}$ de distância.

Os espécimes coletados foram depositados na Coleção Entomológica do Departamento de Sistemática e Ecologia (DSEC) da Universidade Federal da Paraíba. Os dados referentes à precipitação pluviométrica foram obtidos através do Laboratório de Meteorologia, Recursos Hídricos e Sensoriamento Remoto (LMRS) da Universidade Federal de Campina Grande, Paraíba.

\section{Análise de dados}

Para a análise dos dados foi calculado o índice de dominância de Berger-Parker, a equitatividade (MagurRAN 1988), o índice de diversidade de Simpson "unbiased" (D) (Lande et al. 2000) e o índice de diversidade de Shannon-Wiener $\left(\mathrm{H}^{\prime}\right)$ (Magurran 1988) para cada estrato. Foi calculado o índice de correlação não-paramétrico de Spearman (rs) entre a riqueza, a abundância, a diversidade e a precipitação, utilizando o programa Statistica para Windows (versão 5.1). Para comparar o número de indivíduos e de espécies entre os estratos foi utilizado o teste U de Mann-Whitney (Zar 1996).

\section{RESULTADOS}

\section{Riqueza, abundância e diversidade}

Foi coletado um total de 1151 indivíduos pertencentes a 11 espécies nos dois estratos. No estrato de copa, foram coletados 301 indivíduos de três espécies, pertencentes a dois gêneros de Euglossina (Tab. I). Euglossa cordata (Linnaeus, 1789) foi a espécie mais abundante (60,8\% do total da amostra), seguida por Eulaema nigrita Lepeletier, 1741 (38,9\%) e Euglossa perpulchra Moure \& Schlindwein, 2002 (0,3\%). As fragrâncias mais atrativas neste estrato foram: beta ionona (61\% do total) e escatol (38\%).

No estrato de sub-bosque, foram coletados 850 indivíduos pertencentes a 11 espécies e três gêneros de Euglossina (Tab. I). Euglossa cordata foi a espécie mais abundante com 710 indivíduos (83,5\% do total da amostra), seguida por Eulaema nigrita $(8,8 \%)$ e Euglossa perpulchra (3,2\%). A fragrância mais atrativa no estrato de sub-bosque foi: beta ionona ( $84 \%$ do total), seguida por escatol $(7,5 \%)$ e eucaliptol $(4,2 \%)$.

A média mensal do número de espécies capturadas foi significativamente maior no estrato de sub-bosque do que no estrato de copa [Mann-Whitney $Z=3,25$; $p<0,01$ ] (Fig. 1). Não houve diferença significativa no número de indivíduos capturados mensalmente, embora no estrato de sub-bosque a média tenha sido aproximadamente três vezes maior [MannWhitney $Z=1,05 ; p=0,29$ ] (Fig. 2). Não foi observada correlação significativa entre o número de indivíduos ou de espécies e a precipitação pluviométrica. 

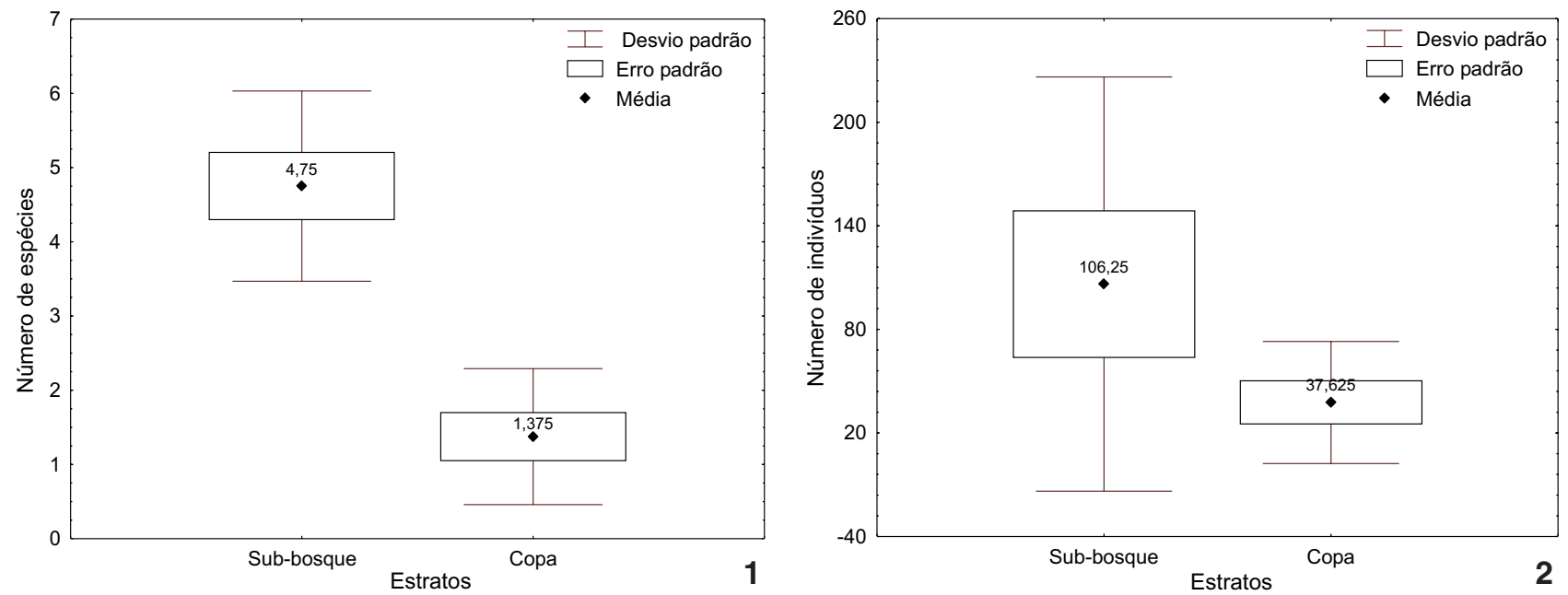

Figuras 1-2. Média mensal do número de espécies (1) e do número de indivíduos (2) nos estratos de sub-bosque e copa da área de Mata Atlântica da Reserva Biológica Guaribas, durante o período de coleta de maio a dezembro de 2002.

Tabela I. Número de indivíduos e espécies de Euglossina nos dois estratos (1,5 m e 10-12m), na área de Mata Atlântica da Reserva Biológica Guaribas, no período de maio a dezembro de 2002. (ST) Sub-total.

\begin{tabular}{|c|c|c|c|c|c|c|c|c|c|c|c|c|c|c|c|c|c|c|}
\hline \multirow{2}{*}{ spécies } & \multicolumn{9}{|c|}{ Sub-bosque $(1,5 \mathrm{~m})$} & \multicolumn{9}{|c|}{ Copa (10-12 m) } \\
\hline & Mai & Jun & & Ago & Set & Out & Nov & Dez & ST & $\mathrm{Ma}$ & Jun & Jul & Ago $s$ & Set O & out $N$ & Vov Dez & & T Total \\
\hline Euglossa cordata (Linnaeus, 1758) & 2 & 23 & 37 & 2672 & 280 & 34 & 58 & & 710 & - & - & - & - & 75 & 50 & 58 & 1183 & 3893 \\
\hline Euglossa frimbriata Rebêlo \& Moure, 1995 & - & - & 1 & - & - & - & - & - & 1 & - & - & - & - & - & - & - & - & - \\
\hline Euglossa ignita Smith, 1874 & 1 & - & - & - & - & - & - & - & 1 & - & - & - & - & - & - & - & - & - \\
\hline Euglossa melanotricha Moure, 1967 & - & - & - & 1 & 1 & - & - & - & 2 & - & - & - & - & - & - & - & - & - \\
\hline $\begin{array}{l}\text { Euglossa perpulchra Moure \& Schlindwein, } \\
2002\end{array}$ & - & & - & - & 22 & 3 & 2 & - & 27 & - & - & - & - & - & - & 1 & - & 1 \\
\hline Eulaema bombiformis (Packard, 1869) & - & - & - & 1 & - & - & - & - & 1 & - & - & - & - & - & - & - & - & - \\
\hline Eulaema cingulata (Fabricius, 1804) & 1 & 6 & 2 & 1 & 1 & 4 & 6 & - & 21 & - & - & - & - & - & - & - & - & 2 \\
\hline Eulaema flavescens (Fri & 5 & - & - & 1 & - & 1 & - & 1 & 8 & - & - & - & - & - & - & - & - & - \\
\hline Eulaema nigrita Lepeletier, 1841 & 2 & 40 & 6 & 4 & 15 & 2 & 3 & 3 & 75 & - & 7 & 67 & 15 & 7 & 3 & 18 & -117 & 7192 \\
\hline Exaerete frontalis (Guérin, 1845) & 1 & - & 1 & - & - & - & - & - & 2 & - & - & - & - & - & - & - & - & - \\
\hline Exaerete smaragdina (Guérin, 1845) & - & - & - & - & - & 2 & - & - & 2 & - & - & - & - & - & - & - & - & 2 \\
\hline Total de indivíduos & 12 & 69 & 47 & 2753 & 319 & 46 & 69 & 13 & 850 & - & 7 & 67 & 15 & 83 & 53 & 77 & 1301 & 011151 \\
\hline Total de espécies & 6 & 3 & 5 & 6 & 5 & 6 & 4 & 3 & 11 & - & 1 & 1 & 2 & 2 & 2 & 3 & 1 & 11 \\
\hline
\end{tabular}

O índice de diversidade (Simpson "unbiased") observado no estrato de copa $(\mathrm{D}=0,48)$ foi maior que no estrato de sub-bosque $(\mathrm{D}=0,29)$. Isso porque a equitatividade foi maior na copa $(J=0,63)$, apesar de terem sido coletadas apenas três espécies, em comparação com o estrato de sub-bosque $(J=0,28)$. Em relação à dominância, o índice de Berger-Parker mostrou que Euglossa cordata foi dominante tanto no sub-bosque quanto na copa $(0,83$ e 0,61 respectivamente). O índice de diversidade $\left(\mathrm{H}^{\prime}\right)$ encontrado na copa $\left(\mathrm{H}^{\prime}=0,99\right)$ foi muito semelhante ao do sub-bosque $\left(\mathrm{H}^{\prime}=0,97\right)$, não apresentando diferenças sig- nificativas, apesar da diferença no número de espécies e indivíduos coletados $(\mathrm{t}=0,28 ; \mathrm{gl}=1131 ; \mathrm{p}<0,01)$.

\section{Atividade mensal das abelhas}

No sub-bosque, os meses de maior abundância foram agosto e setembro. Na copa, não houve um padrão bem definido quanto aos meses de maior abundância, embora o mês de setembro, início da estação seca, tenha sido o que apresentou maior número de indivíduos $(\mathrm{n}=82)$ (Fig. 3).

Com relação às espécies mais abundantes, observou-se 
que Euglossa cordata teve uma maior abundância nos meses de agosto e setembro no sub-bosque, enquanto que na copa a maior abundância ocorreu no mês de setembro. Para Eulaema nigrita, a segunda espécie mais abundante, o maior número de indivíduos ocorreu nos meses de junho na copa e julho no sub-bosque, fim da estação chuvosa.

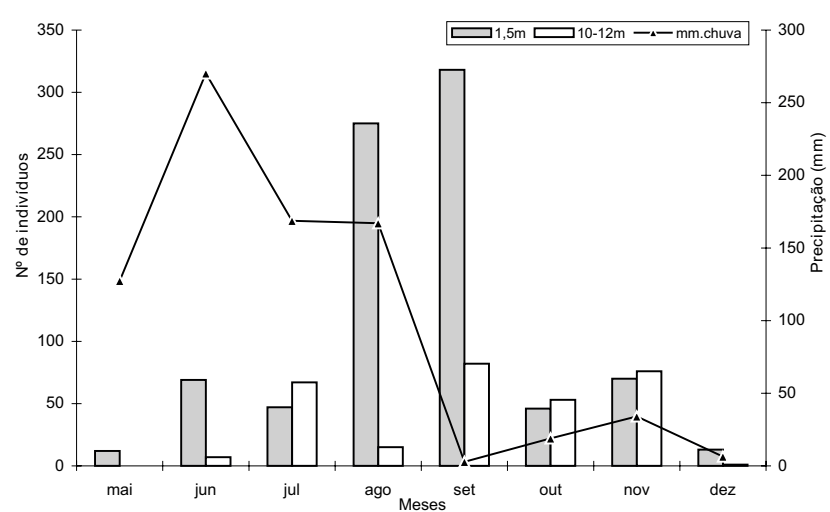

Figura 3. Número de indivíduos de Euglossina capturados em dois estratos na área de Mata Atlântica da Reserva Biológica Guaribas, juntamente com a precipitação pluviométrica, durante o período de maio a dezembro de 2002.

\section{DISCUSSÃO}

\section{Riqueza, abundância e diversidade}

No estudo realizado por RoubIK (1993) a maior abundância e riqueza de Euglossina foram obtidas no estrato de subbosque, enquanto que Oliveira \& Campos (1996) observaram maior diversidade $\left(\mathrm{H}^{\prime}\right)$ no estrato superior e riqueza semelhante nos dois estratos. No presente estudo, o estrato de sub-bosque apresentou uma riqueza significativamente maior em relação à copa. Apesar da diferença não ser estatisticamente significativa, a abundância no sub-bosque foi cerca de três vezes maior. Desse modo, pode-se afirmar que o estrato de sub-bosque foi aquele com maior atividade e riqueza de espécies de Euglossina.

O estudo realizado por MoRato (2001), sobre estratificação da nidificação de vespas e abelhas solitárias, mostrou uma clara estratificação vertical, sendo o estrato superior àquele com maior abundância e riqueza.

Em alguns estudos que investigaram estratificação vertical em Euglossina (Roubik 1993, Oliveira \& CAmpos 1996), não foram observadas grandes diferenças na abundância das abelhas entre os estratos, embora uma grande diferença tenha se mostrado no trabalho de Otero \& SalLenave (2003), quando num total de 88 indivíduos, apenas cinco foram coletados na copa. Porém, como ressaltado pelos próprios autores, seus dados se baseiam em coletas realizadas durante um único mês, não tendo sido consideradas as variáveis sazonais ou fenológicas.
Quanto à diversidade, não houve diferença estatística significativa entre os estratos, como nos estudos de OTERo \& Sallenave (2003) e Roubik (1993). Diversos fatores podem estar relacionados com a estratificação vertical das abelhas, como sazonalidade de recursos e tamanho corporal das espécies (Ramalho 2004), locais para nidificação Morato (2001), termoregulação corporal (MAY \& CASEY 1983) e coloração das abelhas (Otero \& Sallenave 2003). É importante ressaltar que não existem informações sobre a distribuição de ninhos de Euglossina em cavidades naturais nos estratos de florestas. Desse modo, as observações referem-se apenas aos locais de maior atividade de coleta de essências.

A espécie Eulaema nigrita, assim como no presente estudo, foi mais abundante na copa do que no sub-bosque em florestas do Panamá (Roubik 1993). Este fato pode corroborar a hipótese de que Eulaema nigrita é uma espécie típica de áreas abertas ou degradadas (PeruQuetti et al. 1999), visto que as condições existentes no estrato de copa são mais semelhantes às existentes em áreas abertas (Morato 2001). Isto também pode estar relacionado com o fato de que as espécies de Euglossina de grande porte podem suportar uma maior temperatura do que as de pequeno porte, devido a sua maior capacidade de termorregulação (MAY \& CASEY 1983) e, portanto, seriam capazes de ficar mais tempo forrageando no estrato de copa (RoubIK 1993). Outra explicação possível para a estratificação vertical das Euglossina é sugerida por Otero \& Sallenave (2003), de que a menor velocidade do vento, e conseqüente dispersão mais lenta das iscas de cheiro no sub-bosque, propiciariam maior facilidade de detecção das iscas pelas abelhas.

Além disso, a baixa riqueza obtida na copa, comparando com a riqueza e diversidade desse estrato em outros estudos, pode estar relacionada à menor diversidade de Euglossina no ecossistema de Mata Atlântica em relação às florestas tropicais (Bezerra \& Martins 2001) ou ao estado de sucessão ecológica em que se encontra a área de Mata Atlântica da Reserva Biológica Guaribas. Convém lembrar que toda a área da Reserva Biológica Guaribas era um assentamento do INCRA e que a Reserva foi criada há apenas 15 anos, e sua Mata apresenta um dossel de 12 a $15 \mathrm{~m}$.

\section{Atividade mensal das abelhas}

De modo geral, os meses em que as abelhas apresentaram os maiores picos de atividades foram agosto e setembro, início da estação seca. Bezerra \& Martins (2001), em uma mata urbana a cerca de $80 \mathrm{~km}$ da área de estudo, observaram uma maior abundância de Euglossina no meio da estação seca ao início da estação chuvosa. C.F. Martins e colaboradores, coletando em uma mata próxima, na Área de Proteção Ambiental de Mamanguape, no mesmo ano de realização do presente trabalho, também observaram uma maior abundância nos meses mais secos.

No Nordeste do Brasil, de modo geral as Euglossina são mais abundantes na estação seca (verão), o que pode explicar o fato de que Bezerra \& Martins (2001) observaram correlação 
entre a abundância e a temperatura média mensal. Por outro lado, nos estudos realizados em área de Mata Atlântica no sul e sudeste (WitTmann et al. 1989, Rebêlo \& Garófalo 1991, PeruquetTi et al. 1999), houve uma maior abundância e riqueza no período chuvoso que é a estação mais quente do ano.

No presente estudo não foi observada correlação significativa entre a abundância, riqueza e diversidade de Euglossina com a precipitação pluviométrica. Portanto, outros fatores abióticos, como a temperatura (Bezerra \& Martins 2001), e bióticos podem estar influenciando as atividades mensais das espécies (Oliveira 1999). Em adição, o padrão de atividade mensal encontrado pode estar relacionado ao período de floração das espécies vegetais, considerando-se que nas florestas tropicais sazonais o pico de floração, geralmente, ocorre na estação seca e início da estação chuvosa (Frankie et al. 1974).

\section{AGRADECIMENTOS}

Agradecemos ao Dr. Clemens Schlindwein, pela colaboração na identificação das espécies de Euglossa e ao Dr. Marcio Oliveira pelas sugestões ao manuscrito. Ao IBAMA pela licença de coleta concedida. Aos Gerentes da Reserva Biológica Guaribas durante a época de realização do trabalho de campo, Msc. Marcelo Marcelino e Msc. José Carlos de Oliveira e aos funcionários da Reserva pelo apoio logístico. À CAPES pelo apoio financeiro.

\section{REFERÊNCIAS BIBLIOGRÁFICAS}

ACKerman, J.D. 1983. Specificity and mutual dependency of the orchid-euglossine bee interactions. Biological Journal of the Linnean Society, London, 20: 301-314.

Becker, P.; J.S. Moure \& F.J.A. Peralta. 1991. More about Euglossinae bees in amazonian forest fragments. Biotropica, Lawrence, 23 (4b): 586-591.

Bezerra, C.P. \& C.F. Martins. 2001. Diversidade de Euglossinae (Hymenoptera, Apidae) em dois fragmentos de Mata Atlântica localizados na região urbana de João Pessoa, Paraíba, Brasil. Revista Brasileira de Zoologia, Curitiba, 18 (3): 823-825.

Braga, P.I.S. 1976. Atração de abelhas polinizadoras de Orchidaceae com auxilio de iscas-odores na campina, campinarana e floresta tropical úmida da região de Manaus. Ciência e Cultura, Campinas, 28 (7): 767-773.

Braga, P.I.S. 1987. Orquídeas, Biologia floral. Ciência Hoje, Rio de Janeiro, 5 (28): 53-55.

Darrault, R.O. \& C. Schlindwein. 2002. Esfingídeos (Lepidoptera, Sphingidae) no Tabuleiro Paraibano, nordeste do Brasil: Abundância, riqueza e relação com plantas esfingófilas. Revista Brasileira de Zoologia, Curitiba, 19 (2): 429-443.

Dodson, C.H. 1966. Ethology of some bees of the tribe Euglossini (Hymenoptera: Apidae). Journal of Kansas Entomological Society, Kansas, 39: 607-629.

Dressler, R.L. 1982. Biology of the orchid bees (Euglossini). Annual Review of Ecology, Evolution and Systematics, Palo Alto, 13: 373-94.
Eltz, T.; W.M. Whitten; D.W. Roubik \& K.E. Linsenmair. 1999. Fragrance collection, storage, and accumulation by individual male orchid bees. Journal of Chemical Ecology, Syracuse, 25 (1): 157-176.

ERwin, T.L. 1982. Tropical forest: Their richnness in Coleoptera and other arthopods species. The Coleopterists Bulletin, Baton Rouge, 36 (1): 74-75.

Frankie, G.W.; H.G. Baker \& P.A. Opler. 1974. Comparative phenological studies of tree tropical wet and dry forests in the lowlands of Costa Rica. Journal of Ecology, London, 62: 881-919.

Governo do Estado da Paraíba. 1985. Secretaria de Educação. UFPB. Atlas geográfico do Estado da Paraíba. João Pessoa, 160p.

Iвама. 1995. Plano de ação emergencial: Reserva Biológica Guaribas. Brasília, Ministério do Meio Ambiente e Recursos Hídricos e da Amazônia Legal, IBAMA, 66p.

KIMSEY, L.S. 1980. The behavior of male orchid bees (Apidae, Hymenoptera, Insecta) and the question of leks. Animal Behavior, Norway, 28: 996-1004.

KIMSEY, L. S. 1982. Systematics of the genus Eufriesea. University of California Publications in Entomology. Davis, 95: 125.

LANDE, R.; P.J. DeVRIes \& T.R. Walla. 2000. When species accumulation curves intersect: implications for ranking diversity using small simples. Oikos, Lund, 89: 601-605.

MagurRan, A.E. 1988. Ecological diversity and its measurement. Princeton, Princeton University Press, 179p.

May, M.L. \& T.M. Casey. 1983. Thermoregulation and heat exchange in euglossine bees. Physiological Zoology, Chicago, 56 (4): 541-551.

Michener, C. D. 2000. The bees of the world. Baltimore, The Hopkins University Press, 913p.

MMA 2000. Avaliação e ações prioritárias para a conservação da biodiversidade da Mata Atlântica e Campos Sulinos. Ministério do Meio Ambiente. Brasília, Conservation International do Brasil, Fundação SOS Mata Atlântica, Fundação Biodiversitas, Instituto de Pesquisas Ecológicas, Secretaria do Meio Ambiente do Estado de São Paulo, SEDMA/ Instituto Estadual de Floresta Minas Gerais, 40p.

Morato, E.F. 1994. Abundância e riqueza de machos de Euglossini (Hymenoptera: Apidae) em Mata de Terra Firme e áreas de derrubada, nas vizinhanças de Manaus (Brasil). Boletim do Museu Paraense Emílio Goeldi, Série Zoologia, Belém, 10 (1): 95-105.

Morato, E.F. 2001. Efeitos de fragmentação florestal sobre vespas e abelhas solitárias na Amazônia Central. II. Estratificação Vertical. Revista Brasileira de Zoologia, Curitiba, 18 (3): 737-747.

Morato, E.F.; L.A.O. Campos \& J.S. Moure. 1992. Abelhas Euglossinae (Hymenoptera, Apidae) coletadas na Amazônia Central. Revista Brasileira de Entomologia, São Paulo, 36 (4): 767-771.

Oliveira, M.L. 1999. Sazonalidade e horário de atividade de 
abelhas Euglossinae (Hymenoptera, Apidae) em florestas de terra firme na Amazônia Central. Revista Brasileira de Zoologia, Curitiba, 16 (1): 83-90.

Oliveira, M.L. \& L.A.O. Campos. 1996. Preferência por estratos florestais e por substâncias odoríferas em abelhas Euglossinae (Hymenoptera, Apidae). Revista Brasileira de Zoologia, Curitiba, 13 (4): 1075-1085.

Oliveira-Filho, A.T. \& D.A. DE Carvalho. 1993. Florística e fisionomia da vegetação no extremo norte do litoral da Paraíba. Revista Brasileira de Botânica, São Paulo, 16 (1): 115-130.

Otero, J.T. \& A. Sallenave. 2003. Vertical Stratification of Euglossine Bees (Hymenoptera: Apidae) in an Amazonian Forest. Pan-Pacific Entomologist, San Francisco, 79 (2): 151-154.

Peruquetti, R.C. 2000. Function of fragrances collected by Euglossini males (Hymenoptera: Apidae). Entomologia Generalis, Stuttgart, 25 (1): 33-37.

Peruquetti, R.C.; L.A.O. Campos; C.D.P. Coelho; C.V.M. Abrantes \& L.C.O. LisboA. 1999. Abelhas Euglossini (Apidae) de áreas de Mata Atlântica: abundância, riqueza e aspectos biológicos. Revista Brasileira de Zoologia, Curitiba, 16 (Supl. 2): 101-118.

Powell, A.H. \& G.V.N. Powell. 1987. Population dynamics of male Euglossine bees in Amazonian forest fragments. Biotropica, Lawrence, 19 (2): 176-179.

Prates, M.; L.C.S. GatTo \& M.I.P. Costa. 1981. Geomorfologia Projeto RADAMBRASIL, levantamento de recursos naturais. Rio de Janeiro, Ministério das Minas e Energia, vol. 23, p. 301-348.

Ramalho, M. 2004. Stingless bees and mass flowering trees in the canopy of Atlantic Forest: a tight relationship. Acta Botanica Brasílica, São Paulo, 18 (1): 37-47.

RebêLo, J.M.M. 2001. História Natural das Euglossíneas. As abelhas das orquídeas. São Luís, Lithograf, 152p.

Rebêlo, J.M.M. \& C.A. Garófalo. 1991. Diversidade e sazonalidade de machos de Euglossinae (Hymenoptera: Apidae) e preferência por iscas-odores em um fragmento de floresta no sudeste do Brasil. Revista Brasileira de Biologia, Rio de Janeiro, 51: 787-799.

RebêLo, J.M.M. \& C.A. Garófalo. 1997. Comunidade de machos de Euglossini (Hymenoptera: Apidae) em matas semidecíduas do Nordeste do estado de São Paulo. Anais da Sociedade Entomológica Brasileira, Jaboticabal, 26: 243-255.

RebêLo, J.M.M \& F.S. Silva. 1999. Distribuição das abelhas Euglossini (Hymenoptera: Apidae) no estado do Maranhão, Brasil. Anais da Sociedade Entomológica Brasileira, Jaboticabal,
28 (3): 389-401.

Roubik, D.W. 1993. Tropical pollinators in the canopy and understory - Field data and theory for stratum preferences. Journal of Insect Behavior, New York, 6 (6): 659-673.

RoubiK, D.W.; P.E. Hanson. 2004. Abejas de orquídeas de la América tropical: Biología y guía de campo / Orchid bees of tropical America: Biology and field guide. Heredia, Instituto Nacional de Biodiversidad, 370p.

Salmah, S.; T. InOue \& S.F. SaKagami. 1990. An analysis of apid bees richness (Apide) in Central Sumatra, p. 139-174. In: S.F. SAKAgAmi; R. OHgushi \& D.W. RoubiK (Eds). Natural history of social wasps and bees in Equatorial Sumatra. Sapporo, Hokkaido University Press, 274p.

SCHEMSKE, D.W. \& R. LANDE. 1984. Fragrance collection and territorial display in male orchid bees. Animal Behaviour, London, 32: 935-937.

Silveira, F.A \& J.R. Cure. 1993. High-Altitude bee fauna of Southeastern Brazil: Implications for biogeographic patterns (Hymenoptera: Apoidea). Studies Neotropical Fauna Enviroment, Lisse, 28 (1): 47-55.

Silveira, F.A.; G.A.R. Melo \& E.A.B. Almeida. 2002. Abelhas brasileiras: Sistemática e identificação. Belo Horizonte, F.A. Silveira, 253p.

Stern, D.L. 1991. Male territoriality and alternative male behaviors in the euglossine bee, Eulaema meriana. Journal of Kansas Entomological Society, Kansas, 64: 421-437.

Tonhasca, A.; J.L. Blackmer \& G.S. Albuquerque. 2002. Abundance and diversity of euglossine bees in the fragmented landscape of the Brazilian Atlantic forest. Biotropica, Lawrence, 34 (3): 416-422.

Williams, N.H. \& W.M. Whitten. 1983. Orchid floral fragrance and male euglossine bee. Methods and advances in the last sesquidecade. Biological Bulletin, Michigan, 164: 355-395.

Wittmann, D.; M. Hoffmann \& E. Scholz. 1988. Southern distributional limits of euglossine bees in Brazil linked to habitats of the Atlantic and Subtropical rain forest (Hymenoptera: Apidae: Euglossini). Entomologia Generalis, Stuttgart, 14: 53-60

Wittmann, D.; M. Hoffmann \& B. Blochtein. 1989. Seasonality and seasonal changes in preferences for scent (Hymenoptera, Apidae, Euglossini). Entomologia Generalis, Stuttgart, 14 (3/4): 217-221.

ZAR, J. H. 1996. Biostatistical analysis. $3^{\mathrm{a}}$ ed. Prentice-Hall International, Inc., London. 662p.

Recebido em 26.IV.2005; aceito em 04.X.2005. 